\title{
Atuaçáo da Fisioterapia Aquática no Condicionamento Físico do Paciente com AVC
}

\author{
Role of Physical Therapy of Aquatic Fitness In Stroke Patients
}

Fabio Jakaitis ${ }^{1}$, Daniel Gonçalves dos Santos ${ }^{2}$, Carolina Vilela Abrantes',
Silvia Gusman ${ }^{2}$, Simone Cristina Bifulco ${ }^{2}$

\section{RESUMO}

Introduçáo. Acidente vascular cerebral (AVC), segundo a OMS, é uma síndrome de desenvolvimento rápido, com sinais clínicos de distúrbio da função cerebral. Como consequencia, a maioria desses pacientes apresenta uma redução na capacidade aeróbia e força em relação a indivíduos saudáveis. A fisioterapia aquática com os seus efeitos terapêuticos e, princípios físicos e fisiológicos favorecem o controle cardiorrespiratório visando o condicionamento físico nas atividades aeróbicas. Objetivo. Analisar a melhora do condicionamento físico em pacientes com AVC na fisioterapia aquática através dos instrumentos de mensuraçóes: esforço e frequência cardíaca. Método. Foi realizada a avaliação do esforço (teste de Borg) e verificação da frequência cardíaca em 13 pacientes portadores de AVC que realizavam 2 terapias semanais de fisioterapia aquática no setor de hidroterapia do Hospital Israelita Albert Einstein de janeiro a dezembro de 2010. Os pacientes foram avaliados antes, durante e após a sessão de terapia aquática Resultados. Totalizaram 31 testes nos 13 pacientes no período de 12 meses de terapia, sendo observada uma melhora do esforço e frequência cardíaca desses pacientes em 50\%. Conclusáo. Após 6 meses de atividade aeróbica, o esforço e frequência cardíaca dos pacientes foi favorecido. Para comprovações mais significantes, novos estudos serao necessarios com um grupo de paciente maior.

Unitermos. AVC, Hidroterapia, Condicionamento Físico Humano.

Citaçáo. Jakaitis F, Santos DG, Abrantes CV, Gusman S, Bifulco SC. Atuação da Fisioterapia Aquática no Condicionamento Físico do Paciente com AVC.

\begin{abstract}
Introduction. Stroke for World Health Association (WHO) is a syndrome of rapid development, with clinical signs of disturbance of brain function. As consequence, most of these patients have shown a reduction in aerobic capacity and strength compared to healthy subjects. The aquatic therapy, with its therapeutic effects and, physical and physiological principles, may improve the cardio-respiratory system leading to a better physical conditioning. Objective. To evaluate the fitness improvement of stroke patients in aquatic therapy throughout the instruments of metrics: effort and heart rate. Method. 13 stroke patients underwent two weekly treatments of aquatic therapy, hydrotherapy sector of the Hospital Israelita Albert Einstein from January to December 2010. The Borg assessment was performed before, during and after the session of aquatic therapy. Results. We performed 31 tests in 13 pacients within 12 months of therapy, and it was observed an improvement in overall fitness of these patients by $50 \%$. Conclusion. After 6 months later of aerobic activity, there was an improvement of effort and heart rate. Further research are nedeed for more significant evidence.
\end{abstract}

Keywords. Stroke, Hydrotherapy, Physical Fitness.

Citation. Jakaitis F, Santos DG, Abrantes CV, Gusman S, Bifulco SC. Role of Physical Therapy of Aquatic Fitness In Stroke Patients.
Trabalho realizado no Hospital Israelita Albert Einstein - Centro de Reabilitaçáo, Sáo Paulo-SP, Brasil.

1. Fisioterapeuta, mestrando em Reabilitação do Equilíbrio Corporal e Inclusão Social, especialista, Hospital Israelita Albert Einstein São Paulo (SP), professor da Universidade Bandeirante de São Paulo, São Paulo-SP, Brasil.

2. Fisioterapeuta, especialista, Hospital Israelita Albert Einstein, São Paulo-SP, Brasil.
Endereço para correspondência: Fabio Jakaitis

Rua Frei Antonio de Guadalupe, 321 apto 51 - Vila Zelina CEP 03141-070, São Paulo-SP, Brasil. e-mail: fjakaitis@einstein.br 


\section{INTRODUÇÃO}

Segundo dados da OMS, os distúrbios circulatórios estão entre as doenças mais comuns no mundo apresentando altas taxas de mortalidade quando comparadas a outras doenças, sendo o AVC responsável por 30\% dessas mortes ${ }^{1-3}$. Em 1996, a taxa de mortalidade por AVC no Brasil foi de 56,1/100000 habitantes ${ }^{2,3}$.

O AVC é a causa mais frequente de incapacidade neurológica crônica na população adulta e constitui a primeira causa de morte no Brasil ${ }^{4-6}$.

Após o AVC, o indivíduo poderá apresentar alteraçóes neurológicas funcionais relacionadas ao quadro motor, fala e cognição, além de comprometimentos da independência nas atividades da vida diária ${ }^{3,7-9}$.

A redução da capacidade de gerar força, provavelmente é devido à diminuição do recrutamento de unidades motoras durante uma atividade física, redução da capacidade oxidativa dos músculos e uma diminuiçáo global da resistência aeróbia, causando um aumento no gasto energético durante a realização das Atividades de Vida Diária $(\mathrm{AVD})^{3,10,11}$. O alto gasto energético apresentado pelos indivíduos com sequelas pós AVC durante a realização das atividades pode contribuir para a fadiga precoce, dispnéia, depressão, ansiedade, estilo de vida sedentário e consequentemente falta de condicionamento físico $^{3,10,12,13}$.

$\mathrm{Na}$ tentativa de reduzir as taxas de morbidade e mortalidade decorrentes do AVC, nas últimas décadas, uma quantidade considerável de recursos tem sido investida em pesquisa em todo o mundo. O objetivo é de controlar os fatores de risco, a fim de prevenir novos eventos de AVC e cardiovasculares e as complicaçóes decorrentes da inatividade prolongada, aumentando a capacidade aeróbia dos indivíduos ${ }^{3,14-17}$.

Os exercícios terapêuticos em água aquecida atuam nos diversos sistemas do corpo humano ${ }^{15,18,19}$ (Tabela 1). No sistema cardiovascular, há um conjunto de respostas à imersão, incluindo bradicardia, vasoconstrição periférica inicialmente seguida de vasodilatação após alguns minutos imersos e desvio de sangue para as áreas vitais que influenciam na pressão arterial ${ }^{15,20}$.

A melhora cardiovascular apresenta resposta hemodinâmica com redução da pós-carga cardíaca e aumento do bem-estar subjetivo que foram encontrados em pa- cientes com ICC crônica após sauna e banhos quente ${ }^{21-23}$.

Além disso, a melhora da função do endotélio vascular tem sido demonstrada com repetição de terapias em águas quentes em voluntários saudáveis e pacientes cardiopatas ${ }^{21,24,25}$.

Em comparação da imersão com a bipedesteção em solo, o débito cardíaco aumenta de 30 a 32\% no meio líquido, enquanto que em solo ele aumenta $4 \%$ a $5 \%$, associado a uma diminuição de aproximadamente 10 batimentos por minuto em solo ${ }^{26,27}$.

A frequência cardíaca (FC) aumenta com a elevação da temperatura e como resultado do exercício, o aumento é proporcional à temperatura da água e a severidade do exercício ${ }^{15,18}$.

Um dos recursos do tratamento pós AVC é a fisioterapia aquática, devido a facilitaçáo nos movimentos tridimensionais que a água permite em realizar nos pacientes com alta incapacitaçãa ${ }^{26,27}$.

Estudos atuais sobre a fisioterapia aquática no AVC mostra que pacientes com déficit no sistema cardiovascular, podem apresentar melhora devido a facilitação nos exercícios aeróbicos ${ }^{26-28}$.

Portanto, o objetivo desta pesquisa foi de analisar a melhora do condicionamento físico em pacientes com AVC na fisioterapia aquática através dos instrumentos de mensuraçóes: esforço (escala de Borg) e frequência cardíaca.

\section{MÉTODO}

\section{Amostra}

13 pacientes portadores de AVC, que se encontravam em protocolo de atendimento regular com 2 sessóes de fisioterapia aquática semanal no setor de hidroterapia do Hospital Israelita Albert Einstein, foram avaliados de janeiro a dezembro de 2010. Foram incluídos os pacientes portadores de AVC em fase subaguda e crônica de lesão, com cognitivo adequado para entendimento dos comandos solicitados em terapia, com marcha subaquática acima de Functinal Independence Measure (FIM) 4 (apresentando mínimo auxílio nas atividades), e com condições clínicas estáveis para a realização de trabalho aeróbico (avaliação médica). Foram excluídos os pacientes com patologias associadas que comprometam a execução do protocolo, como por exemplo demências, negli- 
Tabela 1

Efeitos fisiológicos da imersão $0^{20}$

\begin{tabular}{|c|c|c|}
\hline $\begin{array}{c}\text { Respostas } \\
\text { Cardiocirculatória } \\
\end{array}$ & $\begin{array}{c}\text { Respostas } \\
\text { Respiratórias }\end{array}$ & Respostasrenais \\
\hline $\begin{array}{l}\text { Aumento retorno venoso } \\
\text { e linfático }\end{array}$ & Aumento do volume central & Aumento da diurese \\
\hline $\begin{array}{l}\text { Aumento do debito } \\
\text { cardíaco e volume cardíaco }\end{array}$ & $\begin{array}{c}\text { Compressão caixa torácica } \\
\text { e abdômen }\end{array}$ & Aumento excreção de sódio \\
\hline Bradicardias & $\begin{array}{l}\text { Aumento do trabalho } \\
\text { respiratório e capacidade vital }\end{array}$ & $\begin{array}{c}\text { Aumento da excreção } \\
\text { de potássio }\end{array}$ \\
\hline $\begin{array}{l}\text { Vasoconstrição } \\
\text { periférica }\end{array}$ & $\begin{array}{l}\text { Aumento do } \mathrm{VO}_{2} \text { max } \\
\text { em água fria }\end{array}$ & $\begin{array}{l}\text { Diminuição da diurese em } \\
\text { água fria com movimento }\end{array}$ \\
\hline
\end{tabular}

gências unilaterais e apraxias.

Todos os pacientes assinaram o termo livre esclarecido para participação e divulgação das informaçóes.

\section{Procedimento}

Após a avaliação inicial, os pacientes incluídos no estudo, iniciaram um protocolo de tratamento direcionado ao condicionamento físico, com verificações dos sinais vitais no início, meio e final da terapia. As avaliaçóes foram realizadas mensalmente pelos autores, totalizando 12 avaliaçôes mensais com aplicabilidade de avaliação antes, durante e após a atividade física. Foi realizada a avaliação do esforço pela escala de Borg, e a FC e a pressão arterial (PA) foi verificada no repouso, acompanhada durante o exercício aeróbico e verificada ao término. $\mathrm{O}$ exercício foi suspenso se a FC cair 10 bpm durante o esforço, conforme sugere a escala de Borg ${ }^{28}$. Como parte integrante das orientaçóes quanto à auto-monitoração, a FC no primeiro minuto da recuperação foi considerada para avaliarmos se a resposta apresentada é normal ou anormal. Espera-se como resposta normal queda acima de $12 \mathrm{bpm}$ para a recuperação ativa e queda acima de $18 \mathrm{bpm}$ para a recuperação passiva ${ }^{29,30}$.

\section{Fluxo do protocolo}

1- A coleta foi realizada somente na primeira terapia mensal do paciente.

2- Pacientes avaliados seguiram critérios de inclusão e exclusão.

3- Todos os pacientes realizaram avaliação inicial para serem incluídos no protocolo da pesquisa onde foram analisadas as condições clínicas, cognitivo e funções motoras dos pacientes.

4- Os sinais vitais (PA e FC) e a escala de BORG foram coletados nos seguintes momentos: a) inicial - após $1 \mathrm{mi-}$ nuto de descanso na borda da piscina; b) médio - após a atividade aeróbica; c) final - término imediato da terapia. 5- A terapia seguiu em 4 etapas: Parte 1: alongamento global; Parte 2: fortalecimento muscular específico e/ou treino de função muscular; Parte 3: condicionamento aeróbico (mínimo de 20 minutos); Parte 4: relaxamento ou finalização de acordo com cada objetivo de terapia.

Obs.: a utilização de carga foi de acordo com a força muscular de cada paciente, onde a carga foi adicionada como progressão da evolução terapêutica do paciente.

\section{Análise estatística}

Para teste de normalidade foi utilizado Teste de Shapiro Wilk, para análise quantitativa foi utilizado teste t-Student, e o teste de Qui-Quadrado para análise qualitativa do Borg. A variação de $\geq$ ou $\leq$ a 1 ou 2 pontos é considerado a melhora ou piora do teste ${ }^{28,29}$.

\section{RESULTADOS}

Finalizou a pesquisa 13 pacientes, 02 pacientes abandonaram por motivos particulares, totalizando 31 testes em um período de 12 meses. No $1^{\circ}$ trimestre pôdese observar que a frequência cardíaca e o teste de esforço (Borg) dos pacientes aumentaram da fase inicial para a fase aeróbica, mas mantiveram alta no final da terapia (aumento de 4 pontos no esforço durante a terapia e diminuição de 2 pontos no final 13 para 17 pontos inicial e 15 pontos no final no Borg e PA foi de 120x80 para 150x90 mmHg) (Gráficos 1 e 2). No 2o trimestre pôde- 
se observar que a Frequência Cardíaca e Borg diminuíram após 6 meses de tratamento (aumento e diminuiçấo de 2 pontos no início, esforço e fim da terapia) e PA foi de 120x80 para 130x80 mmHg reduzindo à normalidade no final da terapia) (Gráficos 3 e 4). No 30 e $4^{\circ}$ trimestre pôde-se observar que não houve mudanças ocorridas nas pontuaçôes quando comparadas aos meses anteriores.

\section{DISCUSSÃO}

Indivíduos que sofreram AVC apresentam necessidade de atividades físicas visando condicionamento físico, devido à inatividades causadas pelas alteraçôes da patologia, podendo interferir diretamente na vida diária destes pacientes e possíveis riscos de novas lesóes musculares e até cerebrais ${ }^{21}$. E este estudo demonstrou que a ativida-

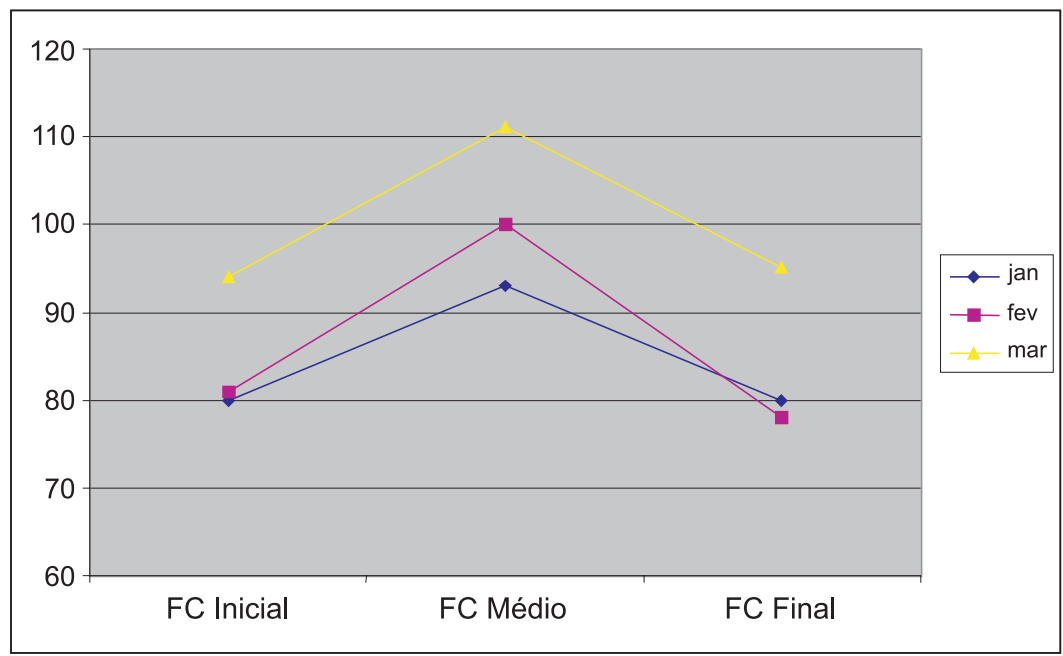

Gráfico 1. Média das frequências cardíacas dos pacientes (1º trimestre).

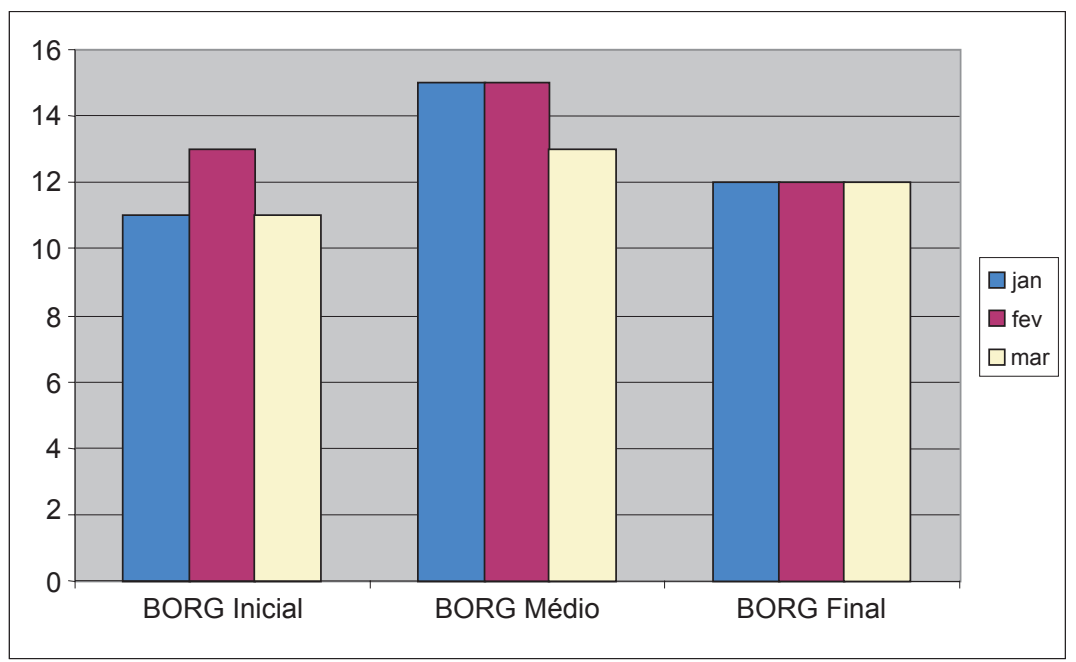

Gráfico 2. Média dos pontos da Escala de Borg dos pacientes (1º trimestre). de aeróbica no meio líquido pode melhorar onde nestes 9 meses observamos uma melhora do condicionamento geral dos pacientes nos primeiros 6 meses, levantando a hipótese que esses indivíduos já condicionaram nos primeiros 6 meses de terapia, levantando a hipótese que o condicionamento físico em pacientes com AVC podem ser alcançados nos primeiros 6 meses de reabilitação ${ }^{29}$. A fisioterapia aquática com os seus efeitos terapêuticos associados aos princípios físicos e fisiológicos, favorece o controle cardiorrespiratório visando o trabalho muscular e condicionamento físico em atividades aeróbicas, vindo de encontro os resultados obtidos neste estudo ${ }^{26}$.

Estudos para prevenção dos riscos do AVC citam a importância da atividade física prolongada, prevenindo os malefícios e inatividade para esses indivíduos, e novamente com este estudo demonstramos que a fisioterapia aquática promove o aumento da capacidade aeróbia ${ }^{21}$.

A reduçáo da capacidade de gerar força provavelmente é devido à diminuição do recrutamento de unidades motoras durante uma atividade física, causando um aumento no gasto energético durante a realização das atividades.

A importância da atividade física realizada com frequência semanal sem interrupçóes para o total aproveitamento fisiológico e ganhos físicos desses pacientes, e no presente estudo observou que em 50\% dos pacientes que não apresentaram melhora, tiveram interrupçóes nas terapias ou alternavam sua frequência de acordo com suas comorbidades e variáveis clínicas ${ }^{3,29,30}$.

As alteraçóes clínicas que surgiram durante a pesquisa, faltas nas terapias e o desligamento do programa, são variáveis importantes que interferiram no condicionamento dos pacientes envolvidos, onde o estudo sugere um numero $(\mathrm{N})$ maior para comprovaçóes mais significantes dos dados aqui citados.

\section{CONCLUSÃO}

$\mathrm{Na}$ população estudada, o efeito do 


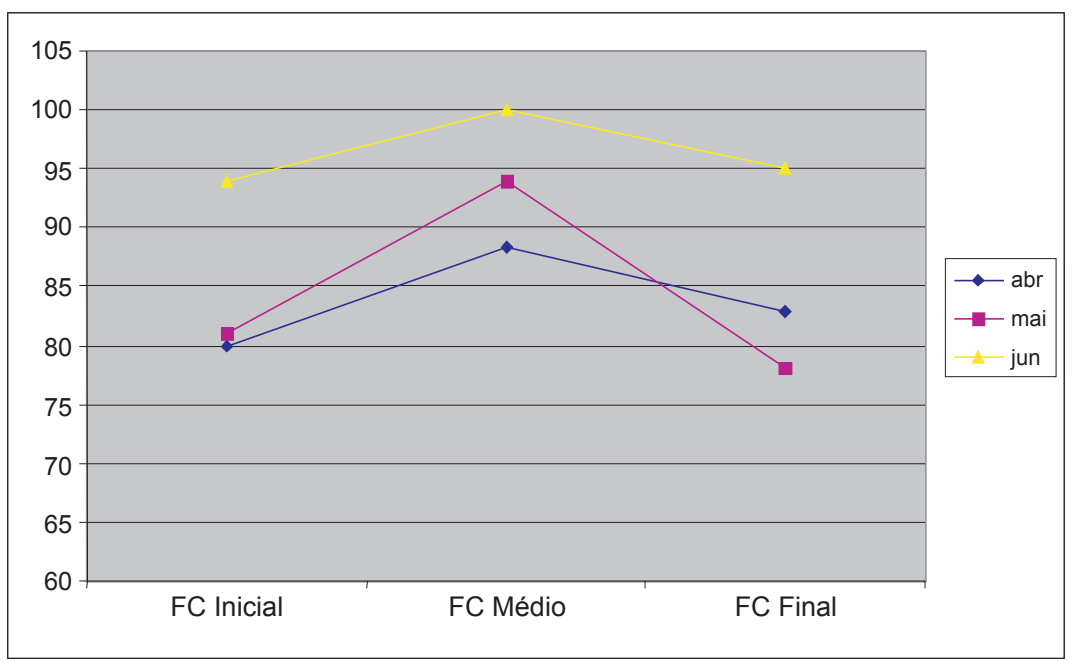

Gráfico 3. Média das frequências cardíacas dos pacientes ( $2^{\circ}$ trimestre).

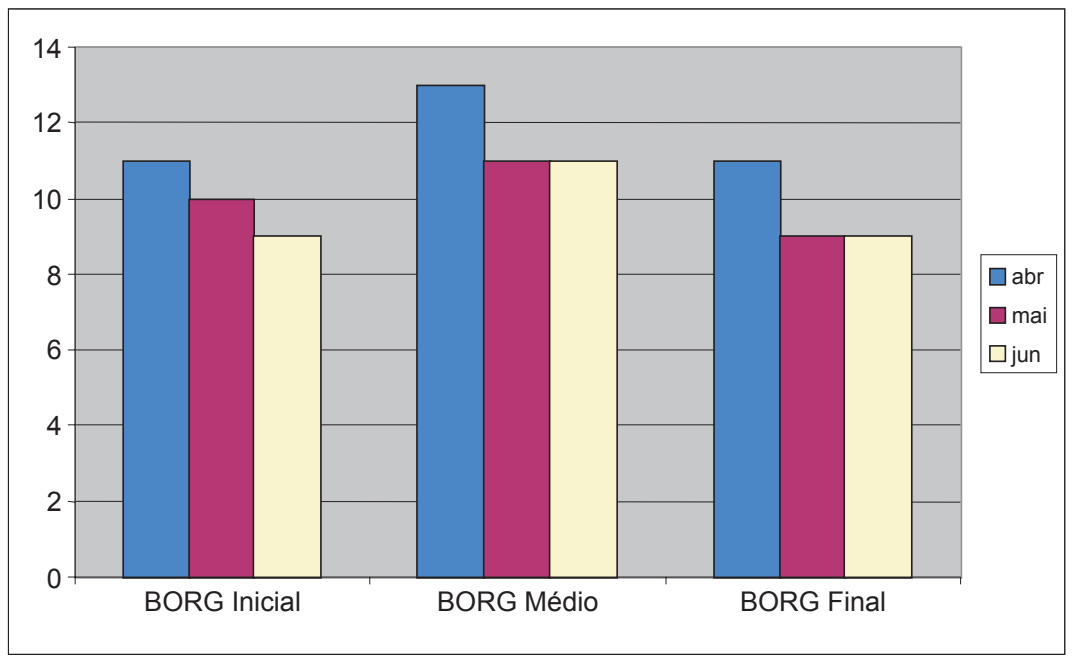

Gráfico 4. Média da Escala de Borg dos pacientes (2º trimestre).

protocolo descrito mostra a melhora do condicionamento físico, podendo ser válido o desenvolvimento de novas pesquisas para comprovação estatística.

\section{REFERÊNCIAS}

1.Wolfe CD. The impact of stroke. Br Med Bull 2000;56:275-86. http://dx.doi.org/10.1258/0007142001903120

2.Sistema de Informaçôes sobre Mortalidade, Ministério da Saúde. Indicadores de Dados Básicos - Brasil 97. Brasília (DF): Ministério da Saúde; 1999, citado em 2005. Disponível em http://www.datasus.com.br.

3.Moura RMF, Lima RCM, Lage DC, Amaral EA A - Efeitos do treinamento aeróbio na qualidade de vida e na capacidade funcional de indivíduos hemiparéticos crônicos Acta Fisiatr 2005;12(3):94-9.
4.Silva VDR, Sant'Ana JE, Vanderlei LCM, Carvalho AC. Análise do comportamento de parâmetros cardiovasculares durante a realização de exercícios físicos em hemiplégicos crônicos Arq Ciênc Saúde 2006;13(4):181-5.

5.Fukujima MM, Martinez TLR. Dislipidemia e acidente vascular cerebral isquêmico. Rev Soc Cardiol Estado de São Paulo 1999;9(4):529-36.

6.Lessa I. Epidemiologia das doenças cerebrovasculares no Brasil. Rev Soc Cardiol Estado de São Paulo 1999;9(4):509-18.

7.Cramer SC. Editorial comment. Spasticity after stroke: what'sthe catch? Stroke 2004;35:139-40.

http://dx.doi.org/10.1161/01. STR.0000105387.38892.98

8.Gordon NF, Gulanick M, Costa F, Fletcher G, Franklin BA, Roth JE, et al. Physical activity and exercise recommendations for stroke survivors; an American Heart Association scientific statement from the Council on Clinical Cardiology, Subcommittee on Exercise, Cardiac Rehabilitation, and Prevention; the Council on Cardiovascular Nursing; the Council on Nutrition, Physical Activity, and Metabolism; and the Stroke Council. Stroke 2004;35:1230-40.

http://dx.doi.org/10.1161/01. STR.0000127303.19261.19

9.Dean CM, Shepherd RB. Task-related training improves performance of seated reaching tasks after stroke: A randomized controlled trial. Stroke 1997;28:722-8.

http://dx.doi.org/10.1161/01.STR.28.4.722

10.Teixeira-Salmela LF, Oliveira ESG, Santana EGS, Resende GP. Fortalecimento muscular e condicionamento físico em hemiplégicos. Acta Fisiatr 2000;7:108-18.

11.Potempa K, Lopez M, Braun LT, Szidon JP, Fogg L, Tincknell T. Physicological outcomes of aerobic exercise training in hemiparetic stroke patients. Stroke 1995;26:101-5. http://dx.doi.org/10.1161/01.STR.26.1.101

12.Monga TN, Deforge DA, Williams J, Wolfe LA. Cardiovascular responses to acute exercise in patients with cerebrovascular accidents. Arch Phys Med Rehabil

1988;69:937-40.

13.Macko RF, Smith GV, Dobrovolny CL, Sorkin JD, Goldberg AP, Silver $\mathrm{KH}$. Treadmill training improves fitness reserve in chronic stroke patients. Arch Phys Med Rehabil. 2001;82:879-84.

http://dx.doi.org/10.1053/apmr.2001.23853

14.Radanovic M. Características do atendimento de pacientes com Acidente Vascular cerebral em hospital, secundário. Arq Neuropsiquiatr 2000;58:99106.

http://dx.doi.org/10.1590/S0004-282X2000000100015

15.Kabuki MT, Sá TS. Os efeitos da hidroterapia na hipertensão arterial e frequência cardíaca em pacientes com AVC. Rev Neurocienc 2007;15:131-4. 16.Lewis RM. Tratado de Neurologia. $10^{\circ}$ ediçāo, Rio de Janeiro: Guanabara Koogan, 2000, 460p.

17.Greenberg DA, Michael JA, Simon RP. Neurologia Clínica. 5 edição, São Paulo: Artmed, 2005, 170p.

18.Souza SEM. Tratamento das doenças neurológicas. Rio de Janeiro: Guanabara Koogan, 2000, 960p.

19.Ekman LL. Neurociência fundamentos para a reabilitação. $2^{\circ}$ ediçâao. Rio de Janeiro: 2004, 477p. 
20.Ruoti RG, Morris DM, Cole AJ. Reabilitação Aquática. São Paulo: Manole, 2000, 463p.

21.Michalsen A, Ludtke R, Buhring M, Spahn G, Langhorst J, Dobos GJ. Thermal hydrotherapy improves quality of life and hemodynamic function in patients with chronic heart failure; Essen and Berlin, German; American Heart Journal 2003;146;6.

http://dx.doi.org/10.1016/S0002-8703(03)00314-4

22.Rowell LB, Brengelmann GL, Blackmon JR, Murray JA. Redistribution of blood flow during sustained high skin temperature in resting man. J Appl Physiol 1970;28:415-20.

23.Chuwa T, Yutaka H, Jong-Chun P, Jin-Won J, Kyoung-Sig C, Yoshihumi T, et al. Acute hemodynamic improvement by thermal vasodilation in congestive heart failure. Circulation 1995;91:2582-90.

24.Kihara T, Biro S, Imamura M, Yoshifuku S, Takasaki K, Ikeda Y, et al. Repeated sauna treatment improvesvascular endothelial and cardiac function in patients with chronic heart failure. J Am Coll Cardiol 2002; 39:754-9.

http://dx.doi.org/10.1016/S0735-1097(01)01824-1
25.Imamura M, Birô S, Kihara T, Yoshifuku S, Takasaki K, Otsuji Y, et al. Repeated thermal therapy improves impaired vascular endothelial function in patients with coronary risk factors. J Am Coll Cardiol 2001;38:1083-8. http://dx.doi.org/10.1016/S0735-1097(01)01467-X

26.Jakaitis F. Reabilitação e Terapia aquática - Aspectos Clínicos e Práticos de Tratamento, São Paulo: editora Roca, 2007, 320p.

27.Caromano F. A.: Candeloro, J. M. Fundamentos da Hidroterapia para Idosos. Arq Ciênc Saúde Unipar 2001;5:187-95.

28.Borg GAV. Psychophysical bases of perceived exertion. Med Sci Sports Exerc 1982;14:377-81.

http://dx.doi.org/10.1249/00005768-198205000-00012

29.Scott,K. Powers \& Edward, T Fisiologia do Exercício. Editora: Manole, 2000, 527p.

30.Nishime EO, Cole CR, Blackstone EH, Pashkow F, Lauer MS. Heart rate recovery and treadmill exercise score as predictors of mortality in patients referred for exercise ECG. JAMA 2000;284:1392-198.

http://dx.doi.org/10.1001/jama.284.11.1392 